\title{
What serious game studios want from ICT research: identifying developers' needs
}

Citation for published version (APA):

Saveski, G. (Author), Westera, W. (Author), Yuan, L. (Author), Fernandez Manjon, B. (Author), Moreno Ger, P. (Author), Stefanov, K. (Author), \& Hollins, P. (Author). (2015). What serious game studios want from ICT research: identifying developers' needs. Digital or Visual Products

Document status and date:

Published: 14/12/2015

Document Version:

Peer reviewed version

Document license:

CC BY-NC-ND

Please check the document version of this publication:

- A submitted manuscript is the version of the article upon submission and before peer-review. There can be important differences between the submitted version and the official published version of record. People interested in the research are advised to contact the author for the final version of the publication, or visit the DOI to the publisher's website.

- The final author version and the galley proof are versions of the publication after peer review.

- The final published version features the final layout of the paper including the volume, issue and page numbers.

Link to publication

\section{General rights}

Copyright and moral rights for the publications made accessible in the public portal are retained by the authors and/or other copyright owners and it is a condition of accessing publications that users recognise and abide by the legal requirements associated with these rights.

- Users may download and print one copy of any publication from the public portal for the purpose of private study or research.

- You may not further distribute the material or use it for any profit-making activity or commercial gain

- You may freely distribute the URL identifying the publication in the public portal.

If the publication is distributed under the terms of Article 25fa of the Dutch Copyright Act, indicated by the "Taverne" license above, please follow below link for the End User Agreement:

https://www.ou.nl/taverne-agreement

Take down policy

If you believe that this document breaches copyright please contact us at:

pure-support@ou.nl

providing details and we will investigate your claim.

Downloaded from https://research.ou.nl/ on date: 26 Apr. 2023 

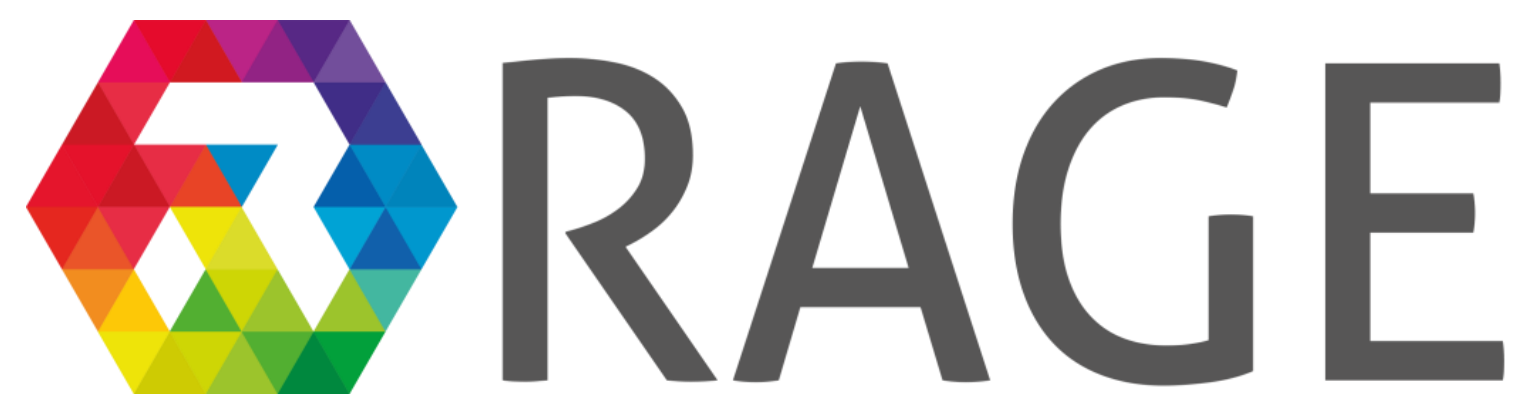

Realising an Applied Gaming Eco-System

\section{What serious game studios want from ICT research: identifying developers' needs}

Grigorij Saveskii, Wim Westera, Li Yuan, Paul Hollins, Baltasar Fernández Manjón, Pablo Moreno Ger, Krassen Stefanov 


\section{Needs analysis:}

"What do you want us to do?"

- "Us" = the RAGE project, offering advanced game technologies

- "You" = (serious) game studios

Structured interviews among European (serious) game studios 


\section{Overview}

1. What is the RAGE project?

2. What do we want to know?

$(90 \%)$

3. What are the outcomes?

$(5 \%)$

$(5 \%)$ 


\section{Overview}

1. What is the RAGE project?

2. What do we want to know?

$(90 \%)$

3. What are the outcomes?

$(5 \%)$

$(5 \%)$ 


\section{The RAGE project}

- H2020 Research and Innovation Action in advanced gaming technologies (ICT-21)

- Partners from

- Games Industry

- Research

- Education and Training

- Business Innovation

$\square$ To support (serious) game studios with new technologies

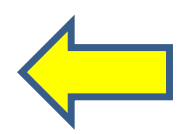




\section{Advanced software modules}

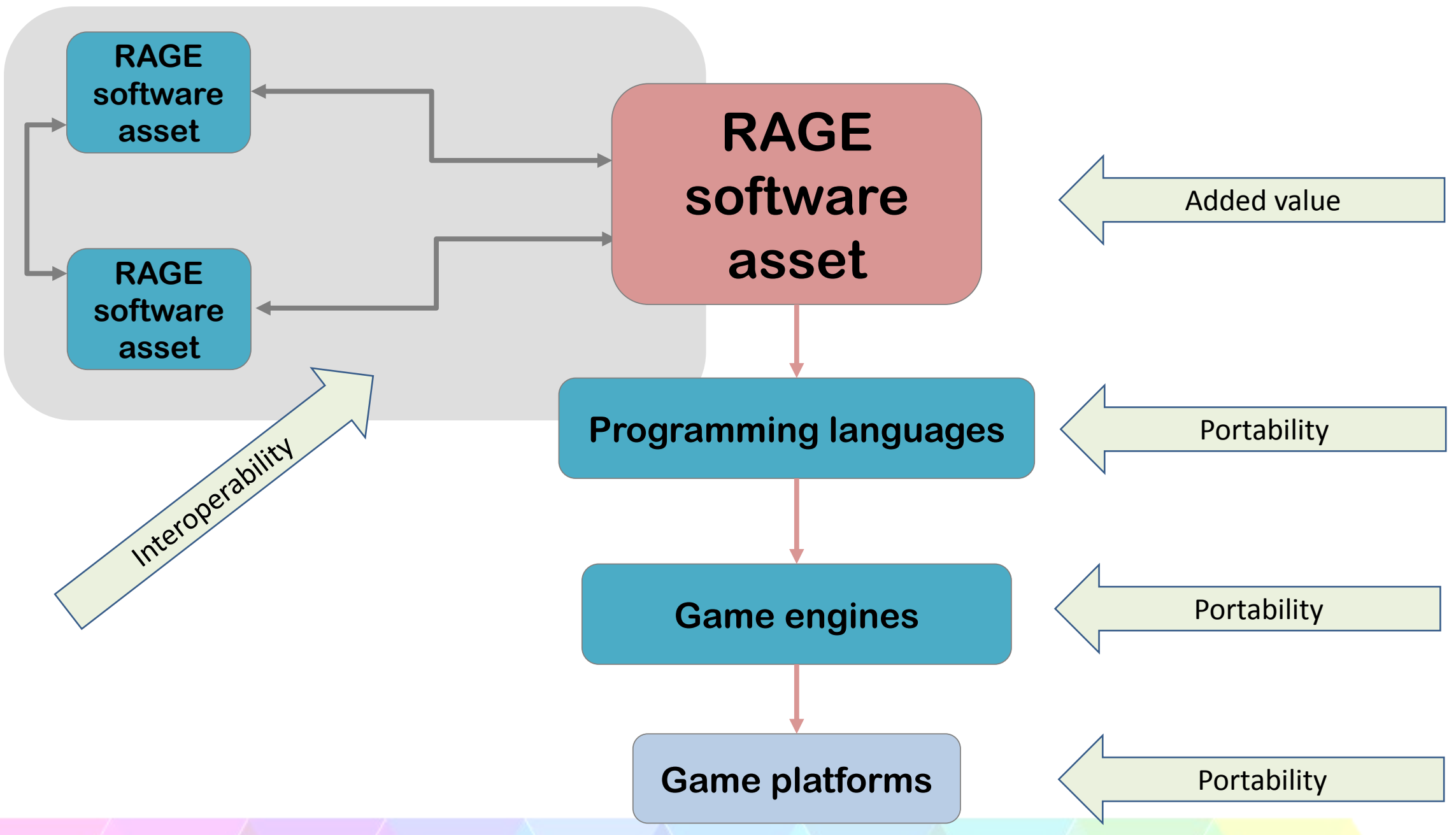




\section{RAGE software asset architecture RAGE}

singletone instantiation

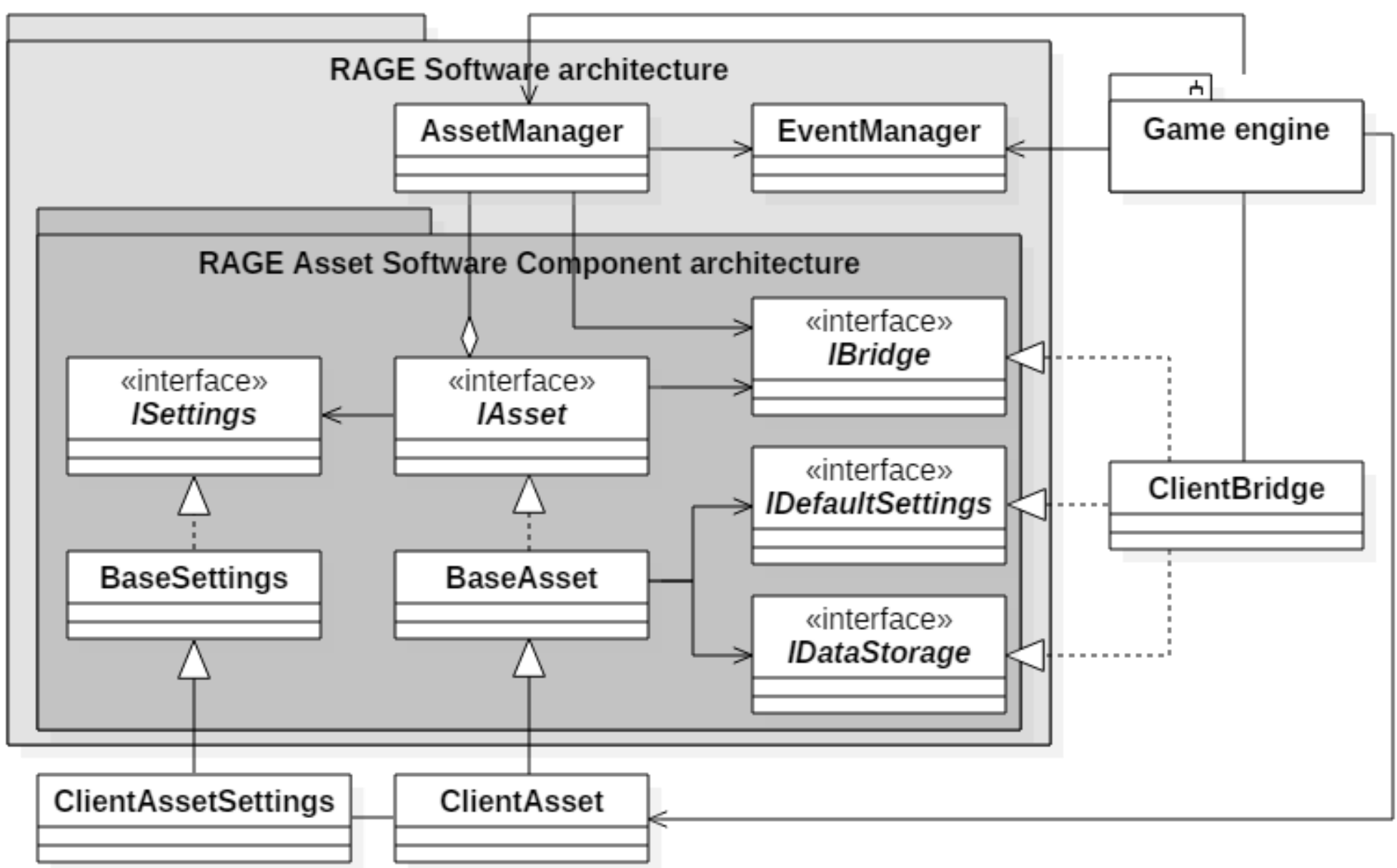




\section{What software assets??}

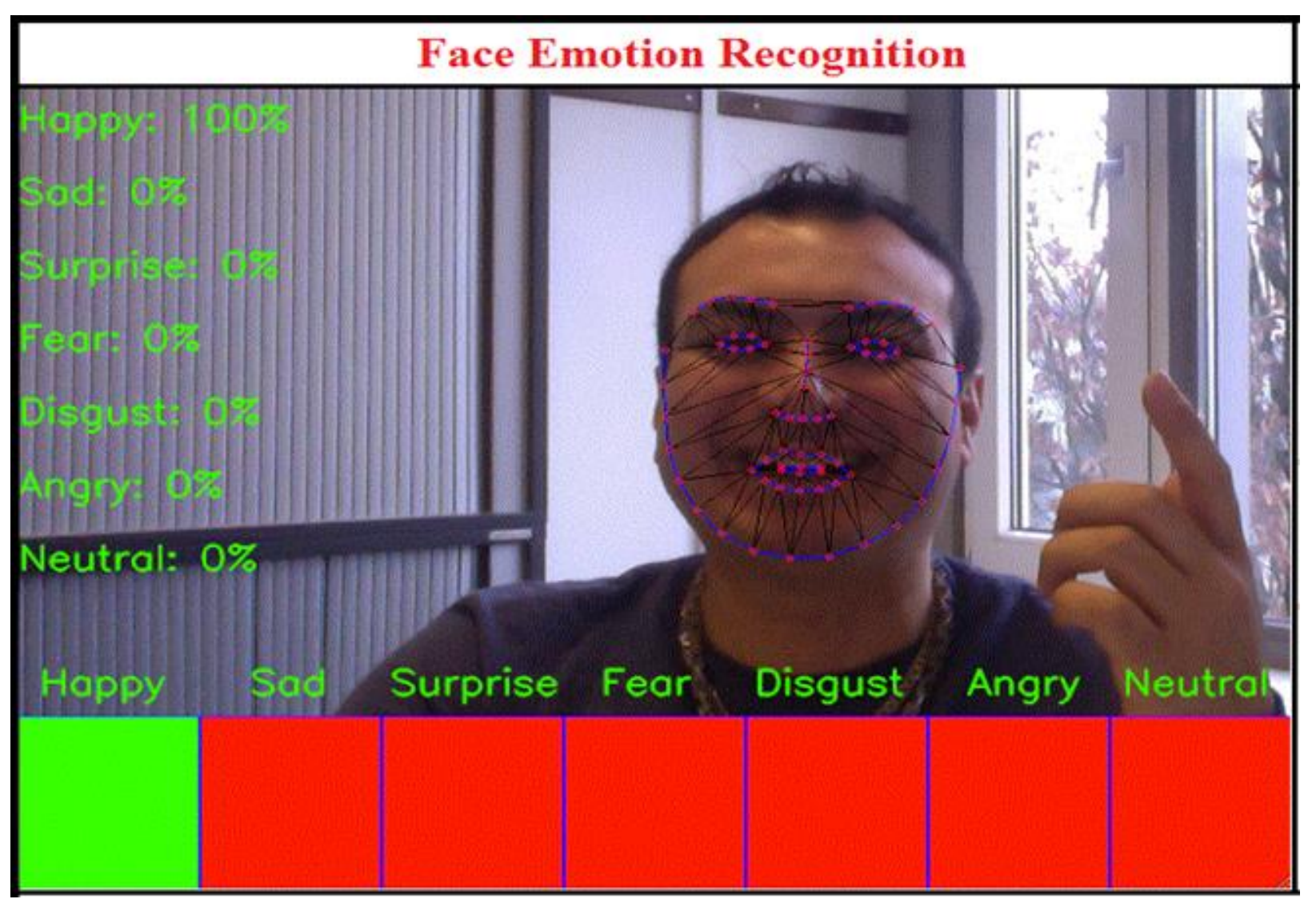




\section{Al reasoning}

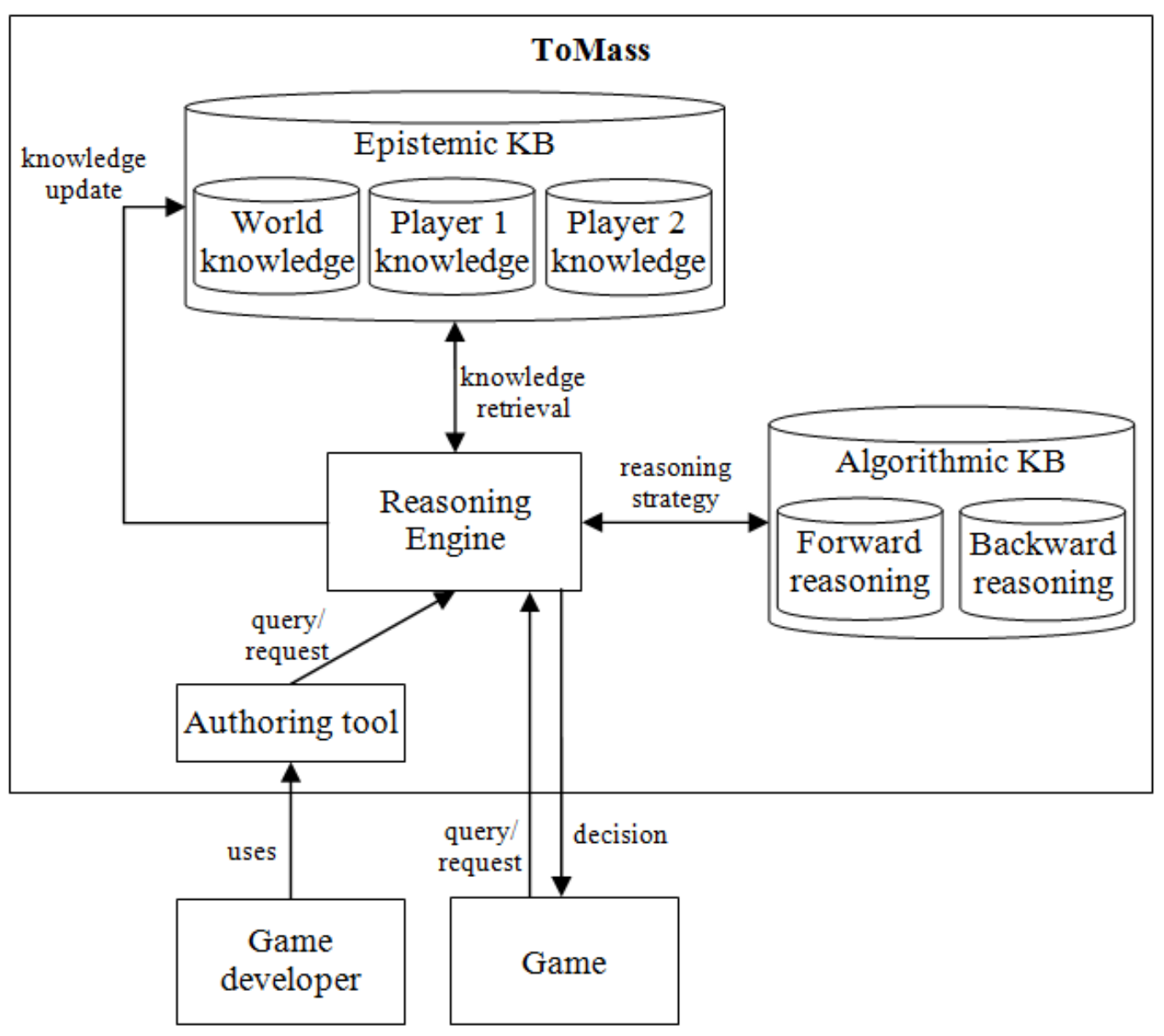




\section{Covert assessment}

\section{(2) RAGE}

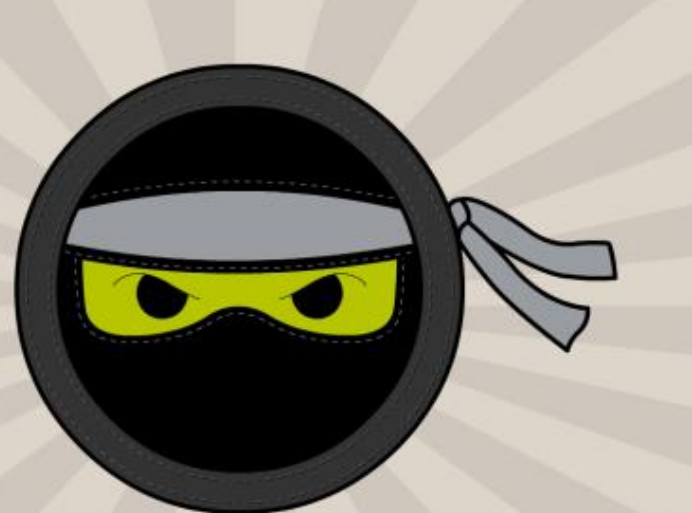

STEALTH ASSESSMENT

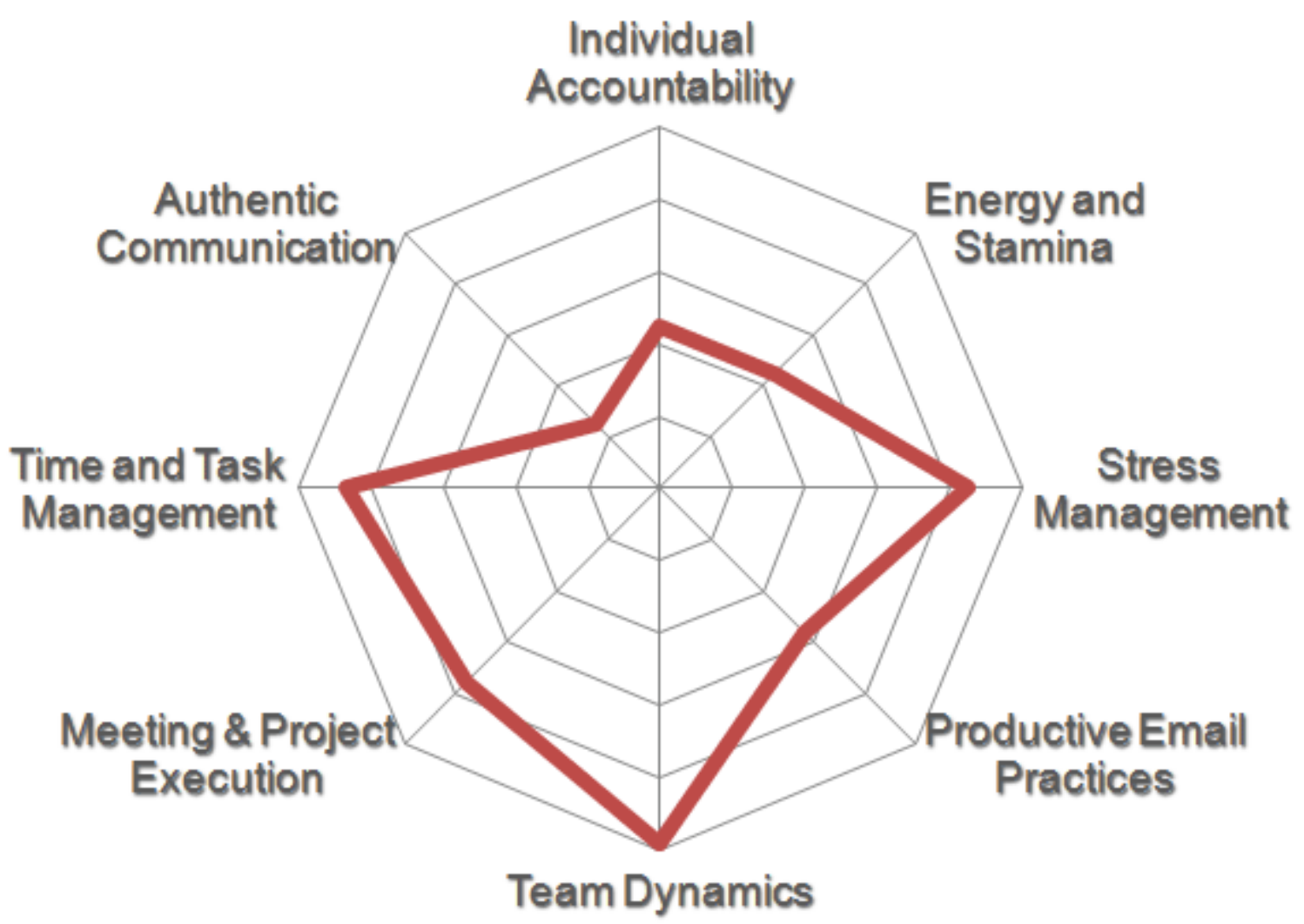

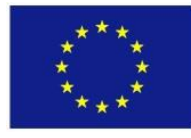




\section{Gaming analytics}

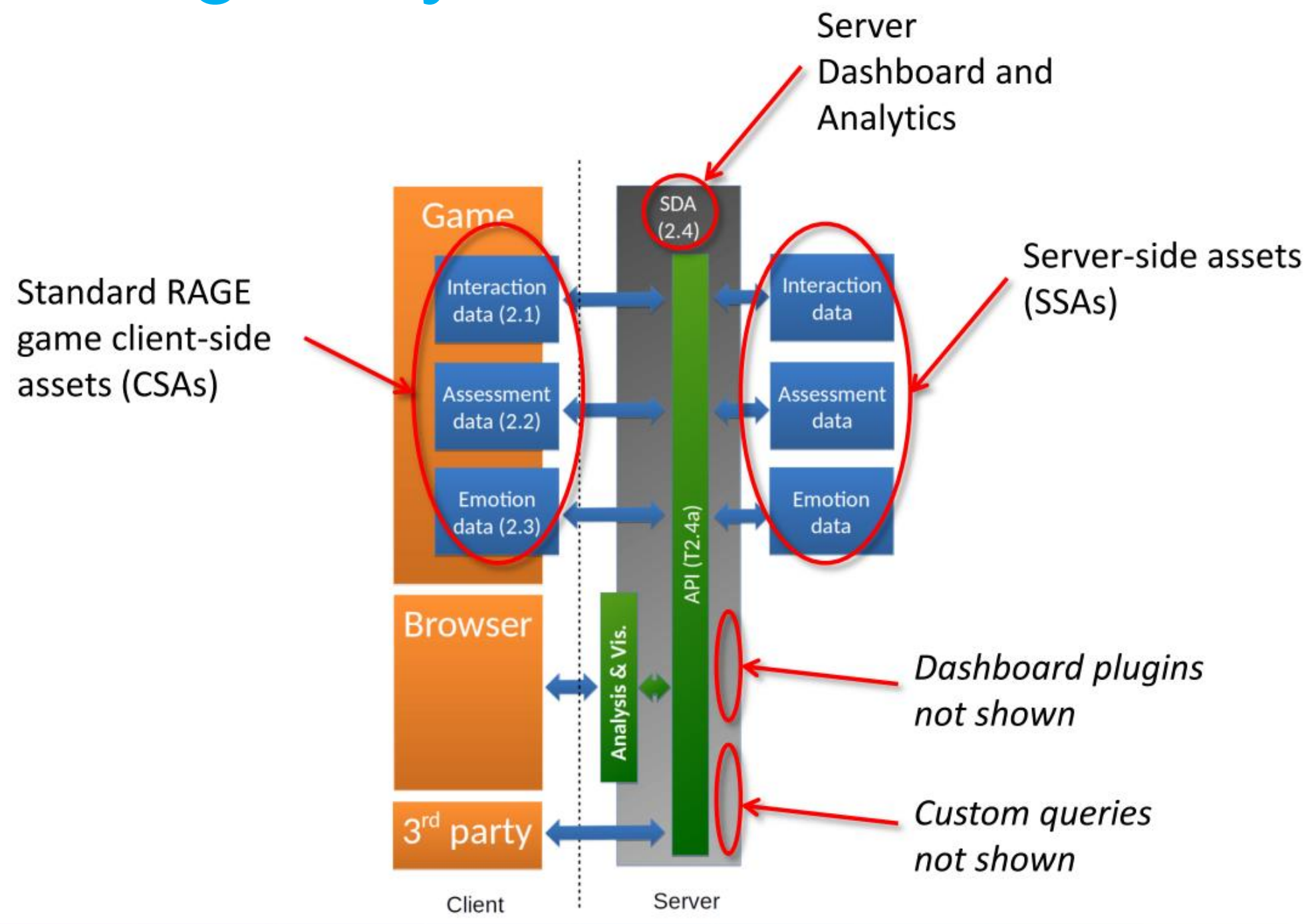




\section{Learning analytics visualisation}

\section{(2) RAGE}

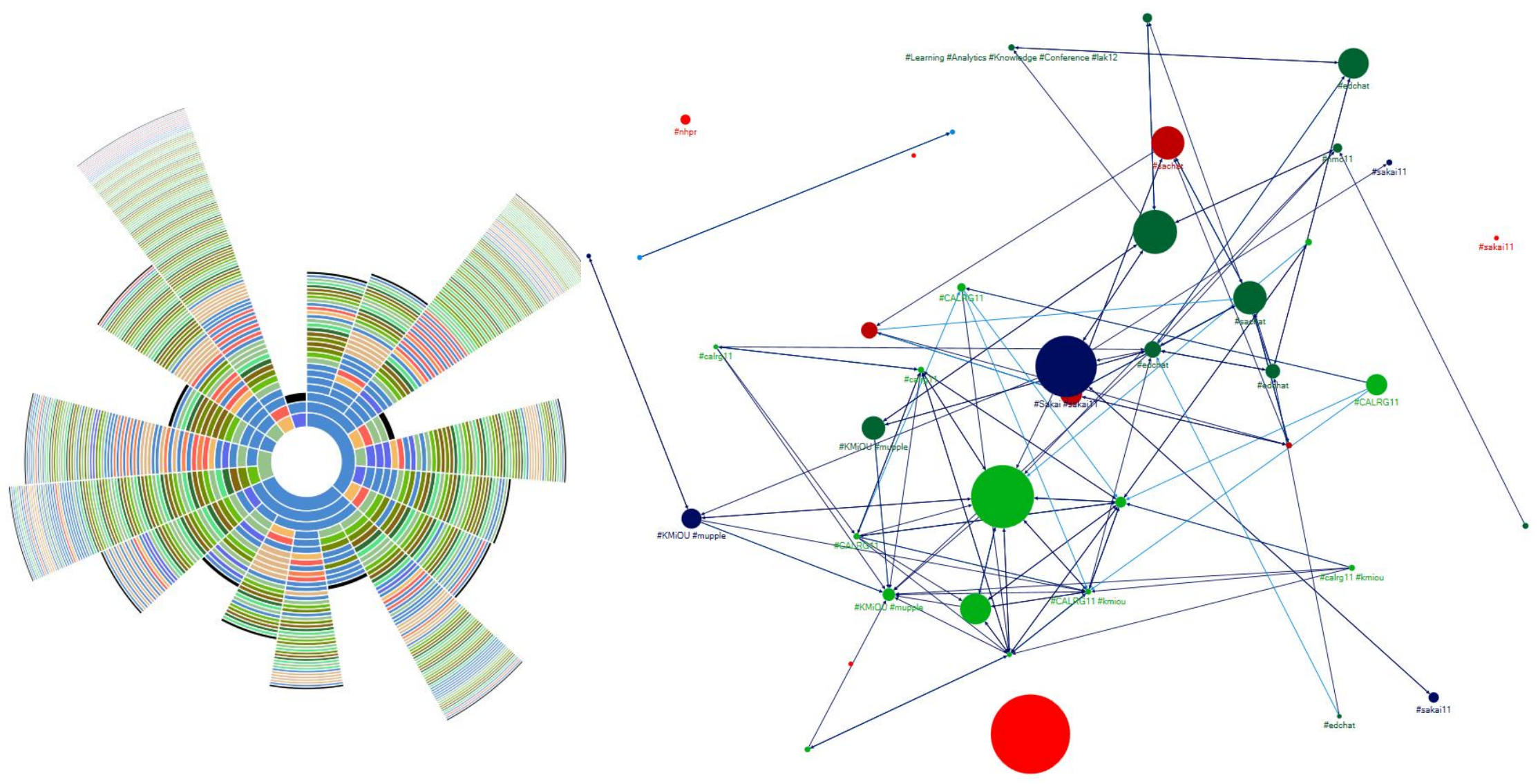




\section{Natural language understanding}

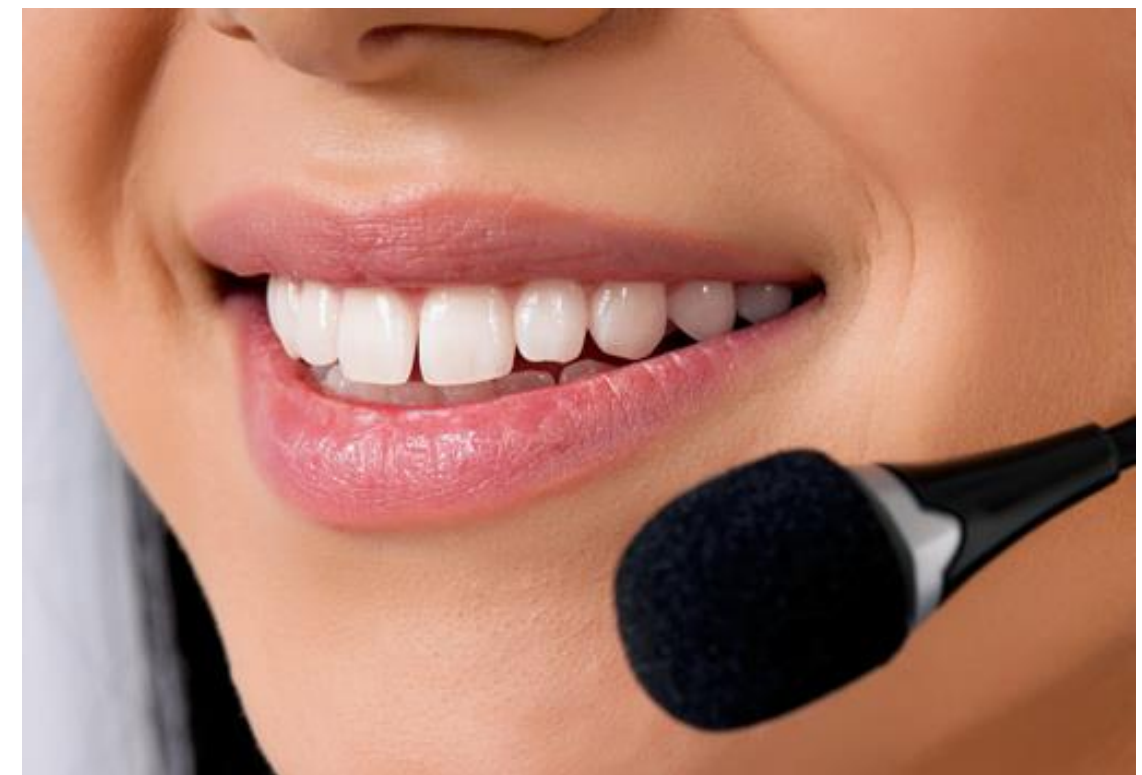

ReaderBench framework

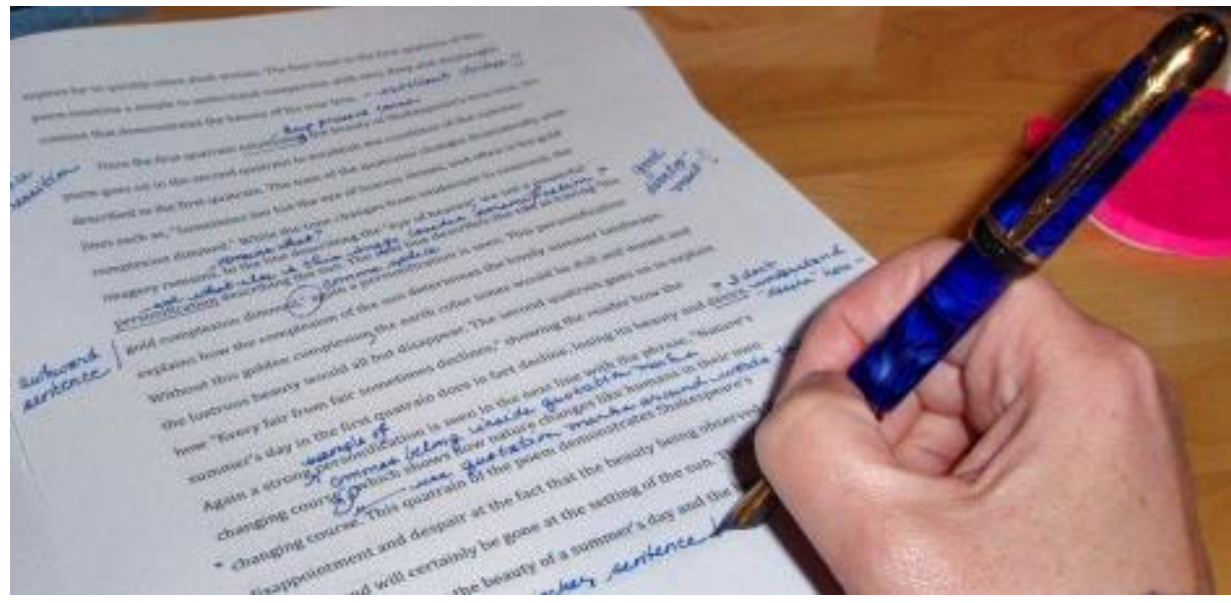




\section{Procedural animation}

\section{(2) RAGE}

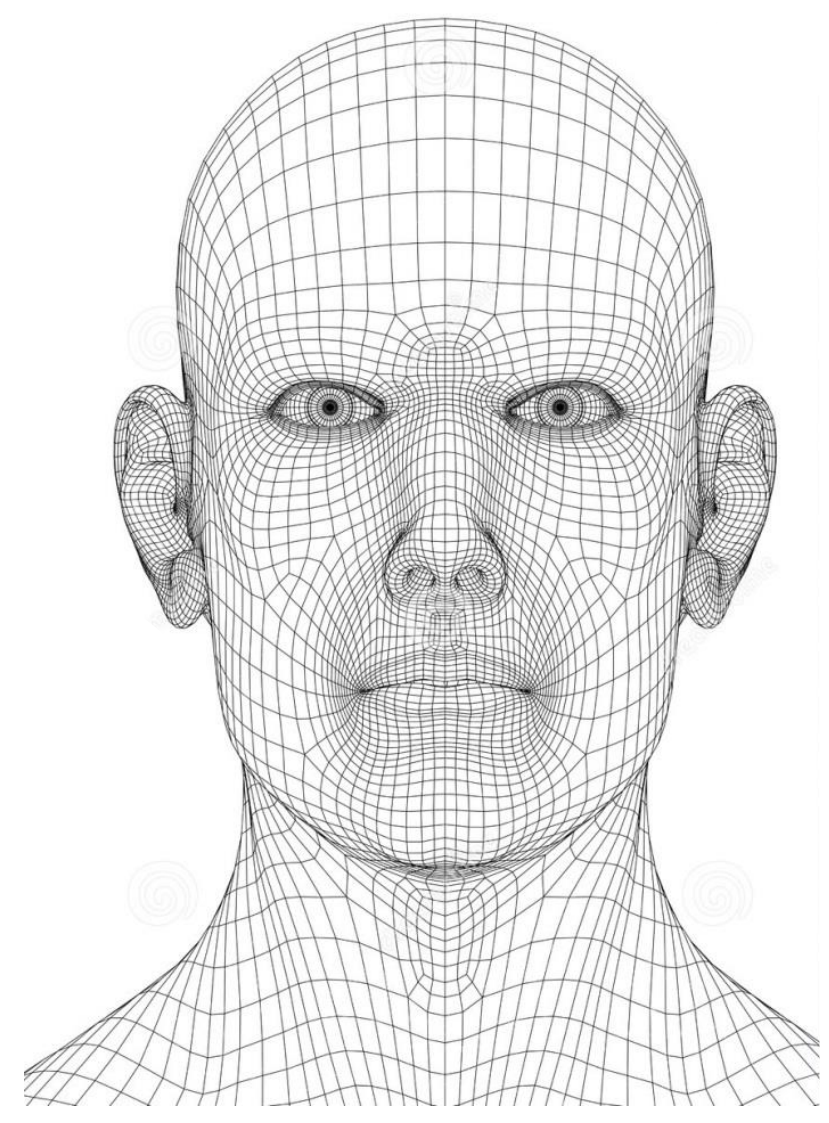




\section{Game adaptation algorithms}

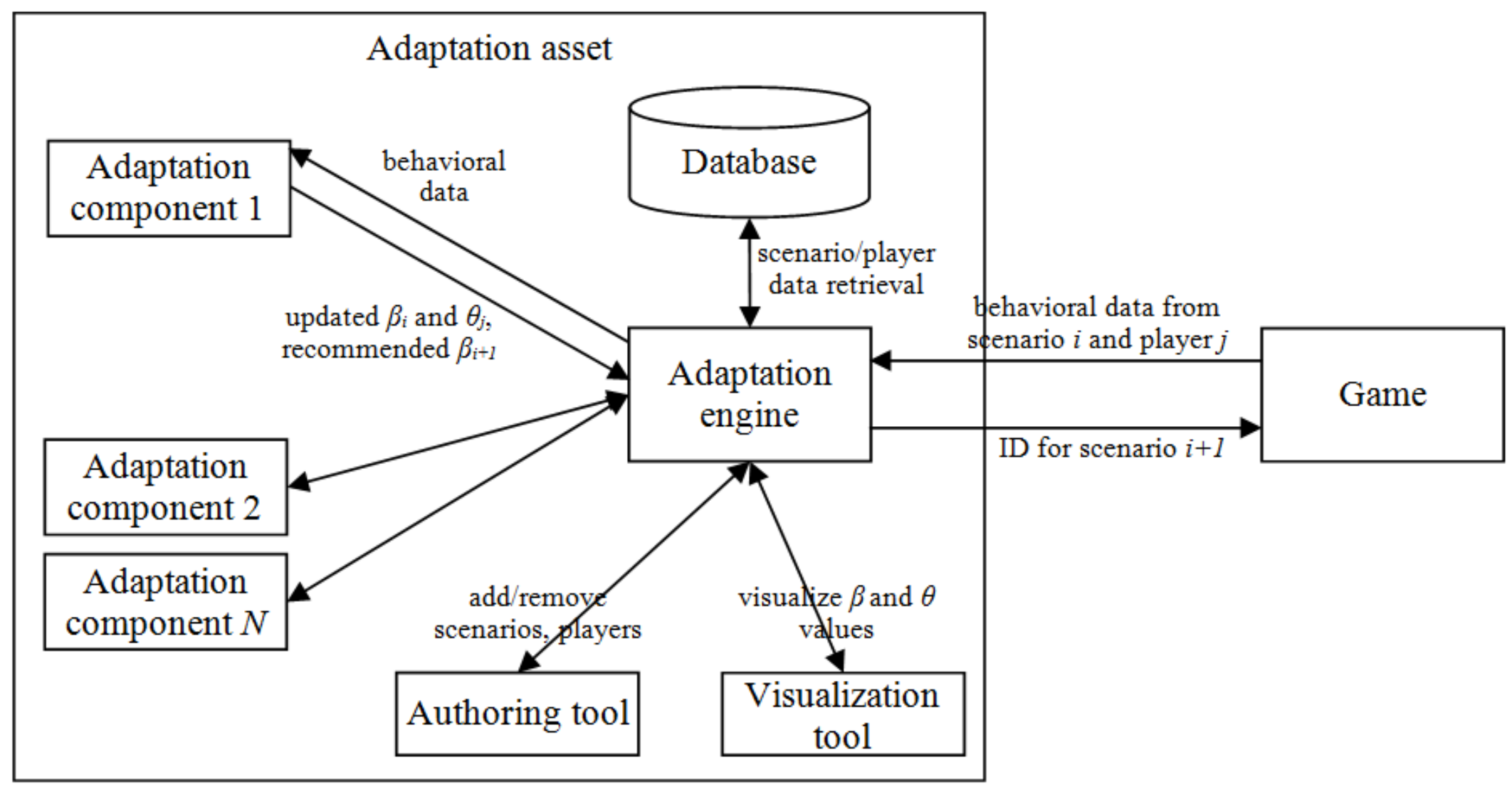




\section{Up to 40 software modules....}

- Social gamification

- Player modelling

- Dialogue authoring

- Multiplayer dialogue analysis

- Emotion appraisal

- Emotions and decision making

- Emotion production

- Identity driven agents

- Competence modelling

- Game evaluation

- Storytelling

$-\ldots$. 


\section{Overview}

1. What is the RAGE project?

2. What do we want to know?

$(90 \%)$

3. What are the outcomes?

$(5 \%)$

$(5 \%)$ 


\section{What do we want to know?}

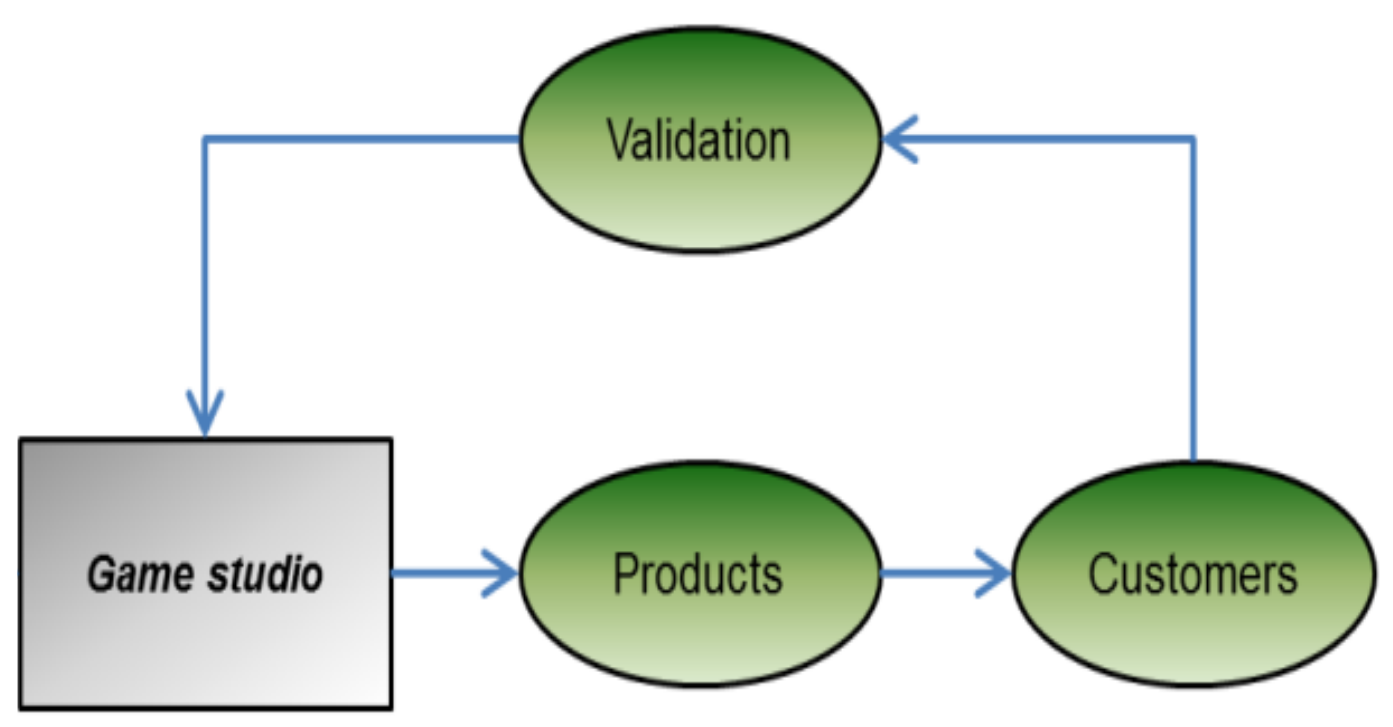




\section{Overview}

1. What is the RAGE project?

2. What do we want to know?

$(90 \%)$

3. Some of the outcomes

$(5 \%)$

$(5 \%)$ 


\section{Technologies used}

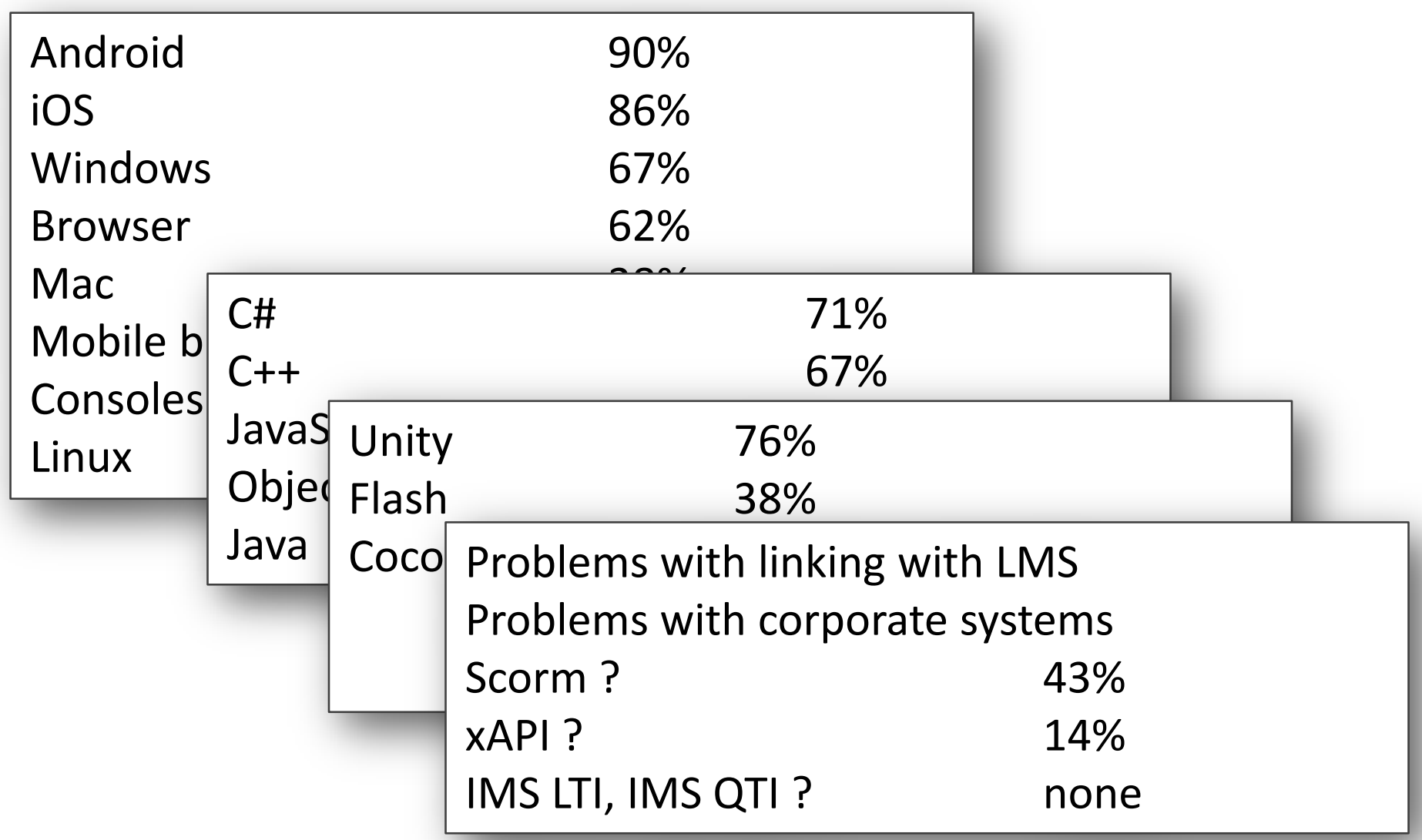




\section{Priorities?}

\section{Emerging technologies}

$-0.7$

$-0.6$

$-0.5$

$-0.4$

$-0.3$

$-0.2$

Unimportant

$-0.1$

Neutral

0.2

0.3




\section{Connect to RAGE}

- Game studios/game developers

- Teachers/educators

- Researchers

- IT developers 


\section{(2) RAGE}

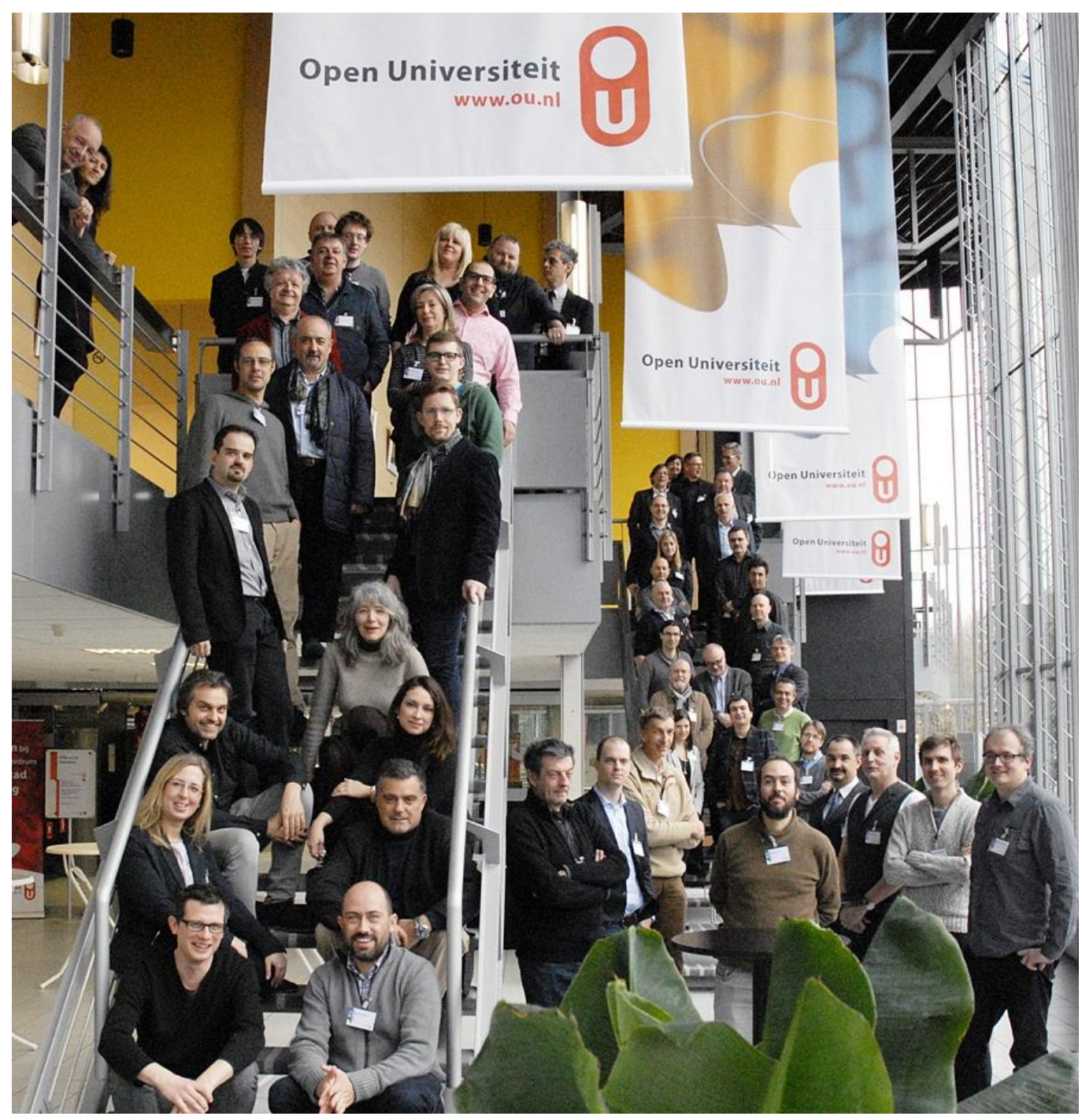

www.rageproject.eu

Wim Westera

Project Coordinator wim.westera@ou.nl

\section{Ruben Riestra}

Innovation Manager ruben.riestra@grupoinmark.com 
